\title{
Diabetic retinopathy and insulin glargine
}

\author{
E. Chantelau
}

Received: 1 July 2009 /Accepted: 9 July 2009 / Published online: 14 August 2009

(C) Springer-Verlag 2009

\section{Abbreviations \\ ETDRS Early Treatment Diabetic Retinopathy Study \\ NPH Neutral protamine Hagedorn}

To the Editor: The recent paper by Rosenstock et al. [1] published in Diabetologia claims that 'this study was specifically designed to detect differences in the incidence of retinopathy progression by fundus photography over a 5 year period' and that it demonstrated 'similar progression of diabetic retinopathy with insulin glargine (A21Gly, B31Arg,B32Arg human insulin) and neutral protamine Hagedorn (NPH) insulin'. The authors explain that, to verify progression status, a side-by-side comparison of baseline and follow-up photographs was conducted for any patient whose Early Treatment Diabetic Retinopathy Study (ETDRS) retinopathy severity scale score progressed by three steps or more, compared with baseline, at any time point during the study. However, Table 1 shows that only 80 out of 513 patients on insulin glargine, and 61 out of 504 patients on $\mathrm{NPH}$, had any diabetic retinopathy at baseline. Among those without retinopathy at baseline, retinopathy can develop but it cannot progress; incidence rates of newly developing retinopathy and progression rates of pre-existing retinopathy must not be confused. In the DCCT study, for instance, the proportion of participants whose retinopathy had progressed by three or more steps at 5 years was approx. 15-35\% (secondary intervention group), which was clearly different from the 5 year incidence of retinopathy (change in ETDRS score of at least three steps from baseline) of approximately $5-15 \%$ in cases without retinopathy at baseline (primary prevention group) [2]. The estimate for power calculations

E. Chantelau $(\square)$

Holthorster Weg 16,

28717 Bremen, Germany

e-mail: lobnig@med.uni-duesseldorf.de by Rosenstock et al. [1], a 20\% 5 year event rate, was taken from the DCCT data [1]. However, the DCCT only included type 1 diabetic patients. In the UK Prospective Diabetes Study on type 2 diabetic patients, cases without pre-existing retinopathy progressed much slower than those with; indeed, the 6 year event rate was less than half that in the DCCT [3]. Hence, the study by Rosenstock and colleagues [1] was considerably underpowered.

Moreover, Table 1 of the paper is unclear. In the category 'baseline ETDRS retinopathy severity', it is stated that 198 patients in the insulin glargine group, and 196 patients in the NPH group had no diabetic retinopathy, leaving 315 and 308 patients with some degree of retinopathy, ranging from microaneurysms only to nonproliferative diabetic retinopathy. However, in the category 'diabetic late complications', it is stated that 80 patients in the insulin glargine group and 61 patients in the NPH group had diabetic retinopathy. This inconsistency needs clarification.

Duality of interest The author declares that there is no duality of interest associated with this manuscript.

\section{References}

1. Rosenstock J, Fonseca V, McGill JB et al (2009) Similar progression of diabetic retinopathy with insulin glargine and neutral protamine Hagedorn (NPH) insulin in patients with type 2 diabetes: a long-term, randomised, open-label study. Diabetologia. doi:10.1007/s00125-009-1415-7

2. The Diabetes Control and Complications Trial Research Group (1993) The effect of intensive treatment of diabetes on the development and progression of long-term complications in insulin-dependent diabetes mellitus. N Engl J Med 329:977-986

3. Kohner EM, Stratton IM, Aldington SJ, Holman RR, Matthews DR, UK Prospective Diabetes Study (UKPDS) Group (2001) Relationship between the severity of retinopathy and progression to photocoagulation in patients with type 2 diabetes mellitus in the UKPDS (UKPDS 52). Diabetic Med 18:178-184 